\title{
Population Approach in Breast Cancer Research Based on Integration of Genetic, Clinicopathological and Genealogical Clues
}

\author{
Sigurdur Ingvarsson ${ }^{*}$ \\ Institute for Experimental Pathology, University of Iceland at Keldur, 112 Reykjavik, Iceland
}

\begin{abstract}
Like other cancer types, breast cancer is considered to be a genetic disease. While the majority of genetic changes are somatic, a minority are in germline. About $10-20 \%$ of breast cancer is thought to be due to a germline mutation in high-penetrance genes, where the major focus has been on BRCA1 and BRCA2. Some of these mutations are defined as founder mutations. Studies on founder mutations yield important information, mainly due to a large number of available carriers with the same mutation, regarding penetrance, expression, genetic modifiers or low-penetrant genes and influence from the environment. Population studies are also valuable due the possibilities for evaluating clinicopathological data in a group of patients who have the same mutation. In Iceland a rare founder mutation has been detected in BRCA1, and a frequent founder mutation has been detected in BRCA2. In addition to population-based studies on genetics and clinicopathology, an extensive analysis of somatic changes in tumours of BRCA2 founder mutation carriers has been made.
\end{abstract}

\section{INTRODUCTION}

Breast cancer has all the hallmarks of a multigenic disease [for review see 1]. Although germline mutations in several genes are well known to be involved in breast tumour progression, this is largely a consequence of somatic evolution. Breast cancer is considered as hereditary if linkage is clear with a relatively highly penetrant gene mutation, while the rest are classified as sporadic. Mutations in BRCA1, BRCA2 and TP53 are highly penetrant, while mutations in others, such as ATM, CHK2 and PTEN, show lower penetrance. Sporadic breast cancer is partly due to the interplay between lowpenetrance genetic factors and exogenous environmental factors. It is roughly estimated that over half of breast cancer is due to the intricate and poorly understood interaction between exogenous environmental factors and multiple low-penetrance genetic factors. No single gene defect has been identified that accounts for the initiation of sporadic breast cancer, and only about $10-15 \%$ of breast cancer patients inherit a familial predisposition. Even in this latter category, only about $50 \%$ of breast cancer can be attributed to the inheritance of mutations in the BRCA1 and BRCA2 suppressor genes. Therefore the underlying etiological bases of most sporadic breast tumours are largely unknown, and additional unidentified genes and the corresponding environmental interactions must play a significant role in the aetiology of breast cancer.

\section{BRCA1 and BRCA2}

BRCA1 is a familial breast- and ovarian-cancer susceptibility gene [2]. Brcal is involved in diverse cellular events and functions, including homologous recom-

*Address correspondence to this author at the Institute for Experimental Pathology, University of Iceland at Keldur, 112 Reykjavik, Iceland;

E-mail: siguring@hi.is bination DNA repair, transcriptional regulation, chromatin remodelling, cell- cycle checkpoint control and ubiquitin ligation [3-6]. BRCA2 is also a familial breast cancer susceptibility gene that is structurally unrelated to BRCA1, but its protein product plays a partial role in the same pathways [7]. The main function of Brca2 is in homologous recombination DNA repair. Both Brca1 and Brca2 bind to Rad51, a protein implicated in recombination and double-stranded DNA repair [8]. The Brca1 and Brca2 proteins participate in the BASC (Brcal associated genome surveillance complex). They are multifunctional proteins involved in complex proteinprotein interactions. The factors binding to Brcal are both specific transcription factors and factors involved in chromatin remodelling. Brca2 is involved in loading of Rad51 to damaged DNA. Mainly active in S and G2 phases of the cell cycle, Brca1 and Brca2 are essential for preserving chromosome structure, suggesting that, in their role as tumour suppressors, they behave as caretakers, suppressing genomic instability. While the role of Brca1 and Brca2 in homologous recombination repair of double-strand DNA breaks is well established, more data are needed to clarify how they act as regulators of cell-cycle events independent of their role in DNA repair.

Even though BRCA1 and BRCA2 are the major genes involved in hereditary breast cancer, they explain only less than $10 \%$ of breast cancers. The majority of breast cancers are believed to be sporadic. In sporadic breast cancer somatic mutations have a major role but it is also influenced by combined effects of low-penetrance sequence variants. The mechanism of BRCA1 or BRCA2 inactivation in tumours is believed to be a double hit, a germline mutation and a somatic deletion $[9,10]$. However, experimental data are lacking to clarify whether losses of the wild-type chromosomes are a prerequisite for non- or abnormal function of the proteins, or whether dominant negative or haplo-insufficient mechanisms can explain the original pathogenesis [11]. Since germline mutations of BRCA1 and BRCA2 are relatively frequent in relation to familial breast cancer, the rarity of somatic 
mutations has been regarded as surprising [12, 13]. This situation is different from the TP53 mutation story, where somatic mutations are relatively common and germline mutations are rare. It is not clear whether some prevention of the molecular mechanism leads to somatic mutations of BRCA1 and BRCA2, or whether the mutations deprive the cells of growth advantage. There could be a particular time frame in normal tissue maturation, after which somatic BRCA1 and BRCA2 mutations are not selected during sporadic breast tumour development, although genesilencing mechanisms and large rearrangements and deletions can influence tumour progression. Even though somatic mutations are rare in BRCA1, it is frequently deleted and expression is decreased in breast tumours, although not always by a known mechanism [14, 15]. Hypermethylations at the promoter region may partly explain the BRCA1 downregulation in sporadic breast tumours $[16,17]$.

\section{Germline (Founder) Mutations in BRCA1 and BRCA2 in the Icelandic Population}

In carriers of BRCA1 and BRCA2 mutations there is a dramatic increase in the risk of breast cancer. Most of the mutations involved are at relatively high penetrance and the majority of women carriers have a lifetime risk of developing breast or ovary carcinoma. Numerous mutations in each gene have been found in most populations studied. The size and number of mutations in these genes have made it difficult to determine their impact on cancer risk. The incidence of mutations in high-risk families varies among different populations. Some populations have a relatively wide spectrum of different mutations, while high-frequency mutations are detected in certain ethnic groups due to a founder effect. When founder mutations have been identified it is possible to examine the prevalence of mutations in different populations and mutation-specific effects on penetrance and disease phenotype.

The Icelandic population originated about 1100 years ago, comprising Nordic and Celtic settlers. The number of primary settlers was small and the population fluctuated over the centuries between 40,000 and 60,000 until the mid19 th century. Several times the population has been adversely affected by cold winters, epidemics and tephra from volcanic eruptions, for instance from the Laki volcano in 1783-4. At that time the population of Iceland fell by $20 \%$, from about 50,000 to 40,000 . Due to improved living standards the population has since risen rapidly, especially in the past 100 years, and the population today is 313,000 . This situation can enhance the probability of founder mutations.

Studies on founder mutations, such as those of Ashkenazi Jewish ancestry and the Icelandic and Polish populations, have made it possible to evaluate the influence of individual BRCA mutations at population level. To date only one mutation in each of the BRCA1 and BRCA2 genes has been identified in the Icelandic population of 313,000 . This is a rare mutation in the BRCA1 gene and a frequent mutation in the BRCA2 gene, both considered to be of founder origin $[18,19]$. The Icelandic BRCA2 founder mutation is present in $8 \%$ of unselected breast cancer patients in Iceland and in $24 \%$ of women diagnosed before the age of 40 years [20]. The BRCA2 999del5 mutation explains $40 \%$ of the increased breast cancer risk in first-degree relatives of Icelandic breast cancer patients [21]. The estimated breast cancer risk in BRCA2 999del carriers at the age of 70 years is about $40 \%$ [22].

\section{Population-Based Studies and BRCA2 999del5 Carriers}

Table 1 summarises the most recent findings on germline mutations in the Icelandic population. Sample collection on Iceland has proven to be an important research tool as it is drawn from population-based series of cancer cases and is linked to genetic and clinical data, as well as data on lifestyle and risk factors [24]. Due to the high frequency of the BRCA2 999del5 mutation, it has received the most attention. The risk of breast cancer in BRCA2 mutation carriers varies from individual to individual, and it appears that the risk has increased in recent generations. These observations imply that non-genetic factors may modify the inherited risk. To date, the factors that appear most strongly to modify the risk include reproductive history and exogenous hormones. Modifying factors include age of menarche, parity, breastfeeding and oophorectomy.

Table 1. Population-Based Studies of Breast Cancer Risk in Icelandic Mutation Carriers

\begin{tabular}{|c|c|c|c|}
\hline Gene & Mutation & Risk/conclusion of study & Reference \\
\hline BRCA1 & D1692N/Splice site* & In $<0.5 \%$ of breast cancer & [18] \\
\hline BRCA2 & $999 \mathrm{del} 5^{*}$ & In $8.5 \%$ of breast cancer, $7.9 \%$ of ovarian cancer, $2.7 \%$ of prostate cancer & [19] \\
\hline “ & “ & In $40 \%$ of male breast cancer & {$[23]$} \\
\hline “ & “ & Variable phenotype & {$[20]$} \\
\hline “ & “ & $37 \%$ risk at age of 70 years & {$[22]$} \\
\hline “ & “ & Risk difference due to reproductive factors & {$[24]$} \\
\hline “ & “ & In $6 \%$ of ovarian cancer, 20 -fold risk & {$[25]$} \\
\hline “ & “ & Quadrupled penetrance over 80 years & {$[26]$} \\
\hline “ & “ & Poor prognosis of prostate patients & {$[27]$} \\
\hline CHK2 & T59K* & Low penetrance, not detected in BRCA2 999del5 & {$[28]$} \\
\hline BARD1 & C557S & Low penetrance, higher frequency in BRCA2 999del5 & {$[29]$} \\
\hline AURKA & F31I & Low penetrance, not detected in BRCA2 999del5 & {$[30]$} \\
\hline
\end{tabular}

*) Founder mutation or mutation only described in Icelanders 
Shortly after the discovery of BRCA1 and BRCA2 several papers published numbers on penetrance and estimated risk of breast cancer and other cancer types. These data were based mainly on families with a high predisposition to breast cancer. Hence mutation penetrance was relatively high, or $70-80 \%$. Also, in these studies different mutations in one of the genes were pooled. Today improved numbers are available on penetrance, based on population studies, including the BRCA2 999del5 Icelandic founder-mutation. The original population studies on the BRCA2 999del5 in the Icelandic population suggested 39\% penetrance [22]. More recent data show that penetrance of the Icelandic BRCA2 founder-mutation has increased about fourfold in 80 years [24]. The cumulative incidence of breast cancer before the age of 70 years in BRCA999del5 carriers was detected as 19\% in 1920 and $72 \%$ in 2002 [24]. Relatively, this is a similar increase in breast cancer risk to that of the general population, so there is a similar overall effect. Possible explanations are changes in lifestyle, involving changes in life expectancy, decline in age of menarche, fewer children born, increased age of mother at birth of first child, menopause at higher age, oral contraceptives etc.

\section{Somatic Events in BRCA2 999del5 Tumours}

Molecular and pathological data suggest a difference not only between BRCA1- and BRCA2-associated tumours, but also between them and sporadic tumours. BRCA1 and BRCA2 tumours are more aggressive than sporadic tumours, as indicated by S-phase, mitosis, aneuploidy, genomic instability and pathological appearance [31]. Other characteristics of BRCA1 tumours are low ER content, elevated lymphocyte infiltration and appearance of medullary phenotype $[32,33]$. The gross genomic instability detected in BRCA1 and BRCA2 tumours fits well with their documented function in DNA repair [34, 35]. Moreover, the chromosome aberration profiles of BRCA1 and BRCA2 tumours differ from each other and from other breast cancers, suggesting that specific genetic pathways operate in the progression of genomic instability in these inherited tumours [34, 35]. Functional support for discrimination between BRCA1, BRCA2 and sporadic breast tumours is also evident from genome-wide gene expression profiles [36].

There seems to be a link between the Brcal and Aurka (Aurora kinase A), since the former is phosphorylated by the latter, an event that is considered to be important for the regulation of the G2-M transition in the cell cycle [37]. It has been shown that Aurka modulates the Brcal inhibition of centrosome function by decreasing the ubiquitin ligase activity of Brca1 [38]. Somatic events in breast tumours of BRCA2 999del5 carriers can include amplified regions where oncogenes are located, as has been shown for the AURKA. While AURKA amplification is found in $22 \%$ of noncarriers it is much more frequent in BRCA2 999del5 carriers, or 70\% [39]. The same study also demonstrates more frequent AURKA amplification if BRCA2 is lost at somatic level. Both AURKA and BRCA2 are involved in maintaining the correct number of centrosomes in the G2-M transition of the cell cycle [40, 41]. It is possible that AURKA amplification increases the risk of tumourigenesis linked to BRCA2 germline mutation through abnormalities in DNA damage response and control of cell division. This may be due to increased risk of AURKA amplification and/or growth selection for AURKA-related pathways leading to tumour formation in BRCA2 mutation carriers.

The majority of TP53 mutations are missense, in contrast to mutations in several other tumour-suppressor genes, where the majority of mutations result in a truncated protein. Some of the TP53 mutations are dominant negative, presumably due to incompetent transcription factor, if one or more mutant copies of the protein are included in the p53 tetrameric form. The germline mutation spectrum is slightly different from the somatic pattern, in line with endogenous mutagenic processes [42]. A high frequency of codon 163 mutation of the TP53 is detected in breast tumours, particularly in a BRCA1 mutational background [43, 44]. The mutation spectrum of TP53 in BRCA1 and BRCA2 carriers is different from that of sporadic tumours, which is consistent with a repair function of Brca1 and Brca2 [44]. The p53 mutants are presumably selected during the malignant progression in the genetic background of BRCA1- and BRCA2-associated tumours. Similarly, elevated somatic mutations of CHK2 have been detected in tumours of BRCA1 genetic mutation carriers [45]. Tumours in BRCA1 carriers have a relatively high frequency of somatic CHK2 mutations, as do tumours in patients with medullary carcinoma [45]. This is of particular interest, since TP53 somatic mutations are also found at a high level in BRCA1 tumours $[43,44]$. These findings of somatic mutations in cell-cycle checkpoint genes such as TP53 and CHK2 are in line with the theory that they increase the rate of tumourigenesis in BRCA1-associated tumours.

It can be hypothesised that in the early stage of BRCA1 and BRCA2 pathogenesis, cells progress through a preliminary crisis phase with massive apoptosis due to accumulation of genetic changes. Further gene alterations, for instance somatic mutations in TP53 or CHK2 or amplification of AURKA, rescue the cell from this senescence phase, and progression is towards reduced apoptosis, enhanced cell growth and a fully malignant phenotype. Even though TP53 mutations are not as frequent in BRCA2- as in BRCA1-associated tumours, overexpression of p53 is detected, suggesting that in BRCA2 mutation carriers the p53 pathway is deregulated by some other mechanisms in addition to mutation [44, 46, 47]. Mouse knockout experiments support the hypothesis of a preliminary crisis phase, and it has been shown that inactivation of $\mathrm{p} 53$, or other checkpoint proteins such as Bub1 and Mad3L, is of importance in tumour progression in mouse cells lacking Brca [48].

The $3 p$ region is not only frequently altered in breast cancer, but is also among the most frequently lost regions in many types of cancer [49]. However, it has been a difficult region in which to find a definite tumour-suppressor gene, and it can be hypothesised that combined functional loss of several tumour suppressor genes located at $3 p$ contributes to tumour pathogenesis. The FHIT gene is located at the most common fragile site in the human genome at 3p14.2, FRA3B, and is frequently altered in breast cancer, particularly if it is of hereditary origin, where BRCA2 is mutated [50, 51]. This could merely reflect the unstable nature of the fragile site in the breast tumour cell, but it is also possible that FHIT plays a 
tumour suppressor role. Specific Fhit pathways have not been identified, but a recent study suggests a role as a transcriptional repressor [52]. The question may be asked, whether the fragile sites in the genome are more sensitive to alterations in a background of germline mutations where DNA repair is dysfunctional. This could be part of the story, but not the only explanation. When comparing losses from chromosomes that carry the most common fragile sites in the genome, FRA3B, FRA16D and FRA6E, only chromosomes $3 p$ and $6 q$ show elevated loss in hereditary tumours associated with DNA repair dysfunction, compared to sporadic breast tumours, but not chromosome $16 q$ [1]. Also, there is higher loss at chromosome $8 p$ in hereditary tumours with mutated repair genes, as against sporadic tumours, even though this chromosome region does not contain a defined fragile site [53].

\section{Low-Penetrance Genes and BRCA2 Mutation Carriers}

Genetic variants of several breast-cancer susceptibility genes have been analysed in BRCA2 999del5 carriers, including BARD1, AURKA and CHK2. Bard1 binds to Brcal and is important for ubiquitin ligase function while Aurka and Chk2 are kinases involved in chromosome segregation and cell-cycle checkpoint, respectively.

Somatic and germline mutations in the CHK2 gene have been described in relation to breast cancer, suggesting that loss of Chk2 is functionally equivalent to TP53 mutations, while mutation frequency is lower in CHK2 than in TP53 [28, 45, 54]. Germline mutations of CHK2 have been found in Li-Fraumeni and Li-Fraumeni-like families, and by population screening of breast cancer patients [28, 45, 54]. The germline variants of $\mathrm{CHK} 2$ analysed so far by population screening seem to be lowpenetrance alleles conferring susceptibility to breast cancer $[28,55]$. Population-based analysis of a mutation that abolishes kinase activity indicated a $5 \%$ frequency in individuals with breast cancer, and a twofold and tenfold increased risk of breast cancer in females and males respectively [55].

It has been suggested that Brca1-dependent ubiquitination activity in concert with Bard1 marks the centrosomes, and inhibits their reduplication [56, 57]. Mutations of BARD1 are found at low frequency in breast cancer [58, 59]. The role of Bard1 in the Brca1 and Brca2 pathways and genomic stability is further established in knockout mouse experiments [60]. The BARD1 C557S genetic variant is detected in the European population and in Americans of European origin. One population-based cohort of 1,090 Icelandic breast-cancer patients and 703 controls suggest that there is a minor elevation in risk of breast cancer in BARD1 C557S carriers, which is further elevated in BRCA2 999del5 carriers [29]. This is the opposite of the low penetrance alleles of $\mathrm{CHK} 2$ and AURKA, T59K and F31I respectively. In both cases there is an increase risk of breast cancer in carriers, with the exception of BRCA2 999del5 carriers [28, 30]. Therefore it is clear that it is important, when looking for lowpenetrance cancer-susceptibility genes, to acknowledge the influence of major cancer genes such as BRCA1 and BRCA2. Likewise, it is important to know the status of low-penetrance genes when estimating the penetrance of BRCA1 and BRCA2.

A possible explanation could be in the progression of breast tumours in BRCA2 carriers. Presumably there is a preliminary phase with apoptosis and senescence, due to failure of DNA repair, resulting in less viable cells (Fig. 1). Somatic events could influence the progression to cancer as described earlier, but this could also be influenced by genetic background or low penetrance genes. Accordingly, the given AURKA or CHK2 variants could entail less growth advantage and BARD1 increased growth advantage, influencing cancer progression (Fig. 1).

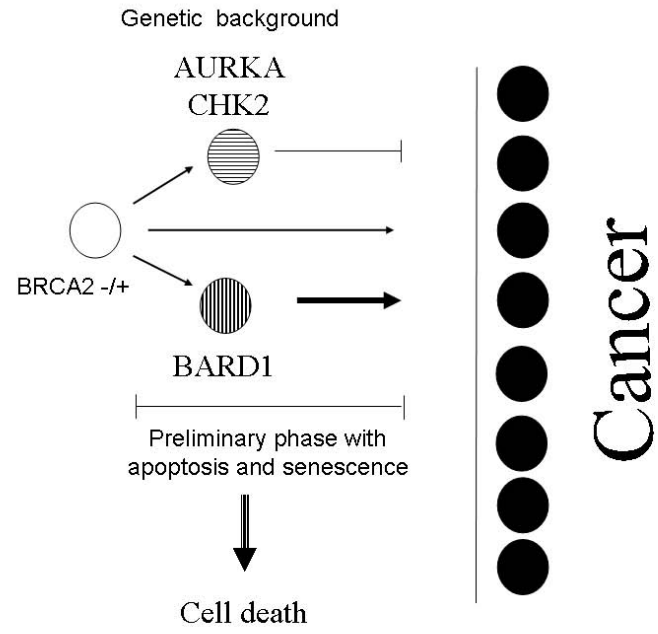

Fig. (1). Theoretical scheme of breast cancer progression in individuals carrying BRCA2 germline mutation. Presumably a BRCA2 defect leads to a preliminary phase with induction of genomic instability and activation of cell-cycle checkpoints and apoptosis. Gene variants in other genes, such as AURKA, CHK2 and BARD1, could either enhance this preliminary phase resulting in reduced cell viability or rescue the cells from checkpoint control and apoptosis phase, resulting in growth advantage for the breast tumour cell, depending on which gene is involved and the variant type (see main text for references and details).

\section{CONCLUSION}

Genetic background is of importance for breast-cancer development, and gene variants are many and have both high and low penetrance. As a significant proportion of sporadic breast cancer can be explained by interaction between lowpenetrance genetic factors and exogenous environmental factors, further investigation of the genes in the pathways initiated by DNA-damaging, mutagenic environmental agents is important. There is strong evidence that genomic instability has a role in breast cancer pathogenesis, particularly in hereditary breast cancer, and possibly a role in sensitivity and resistance to therapy. Cells with elevated genomic instability are viable due to selective pressure of genes involved in cell turnover. It seems clear that somatic events in tumours of individuals with germline mutation in breast-cancer-predisposing genes are fundamental for breast-tumour pathogenesis. In carriers of highly penetrant genes such as BRCA1 or BRCA2, caution must be used in extrapolating the data to the general population, and also to other locations in the 
corresponding genes. Founder mutations permit analysis of a large number of cases, and provide more accurate information on penetrance, expression, and genetic and environmental modifiers of risk. This information can be useful in understanding the role played by these genes in the incidence of breast cancer, in order to target genetic testing, provide individual risk assessment, and design better therapeutic strategies. The evidence of differences in susceptibility and in age of onset among carriers of a specific mutation makes it easier to define the role and importance of risk-modifying factors, leading to improved disease management. Information on new breast cancer genes is expected in the near future, and genetic association studies, which survey the entire genome, are being developed for uncovering the genetic basis of breast cancer. Such studies have identified several novel loci, including common variants on chromosomes 2 and 16 [61]. New information is currently under consideration for developing therapy strategies in hereditary breast cancer. This information also includes the somatic events in hereditary breast cancer. One example of a relevant question to be addressed is whether breast tumours with mutations in BRCA2 could be promising candidates for Aurka-targeted treatment.

\section{REFERENCES}

[1] Ingvarsson S. Genetics of breast cancer. Drugs Today 2004; 40: 991-1002.

[2] Miki Y, Swensen J, Shattuck-Eidens D, et al. A strong candidate for the breast and ovarian cancer susceptibility gene BRCA1. Science 1994; 266: 66-71.

[3] Moynahan ME, Chiu JW, Koller BH, Jasin M. Brca1 controls homology-directed DNA repair. Mol Cell 1999; 4: 511-8.

[4] Welcsh PL, Lee MK, Gonzalez-Hernandez RM, et al. BRCA1 transcriptionally regulates genes involved in breast tumorigenesis. Proc Natl Acad Sci USA 2002; 99: 7560-5.

[5] Bochar DA, Wang L, Beniya H, et al. BRCA1 is associated with a human SWI/SNF-related complex: linking chromatin remodeling to breast cancer. Cell 2000; 102: 257-65.

[6] Baer R, Ludwig T. The BRCA1/BARD1 heterodimer, a tumor suppressor complex with ubiquitin E3 ligase activity. Curr Opin Genet Dev 2002; 12: 86-91.

[7] Wooster R, Bignell G, Lancaster J, et al. Identification of the breast cancer susceptibility gene BRCA2. Nature 1995; 378: 789-92.

[8] Chen JJ, Silver D, Cantor S, et al. BRCA1, BRCA2, and Rad51 operate in a common DNA damage response pathway. Cancer Res 1999; 59: 1752S-56S.

[9] Smith SA, Easton DF, Evans DG, Ponder BA. Allele losses in the region $17 \mathrm{q} 12-21$ in familial breast and ovarian cancer involve the wild-type chromosome. Nat Genet 1992; 2: 128-31.

[10] Gudmundsson J, Johannesdottir G, Bergthorsson JT, et al. Different tumor types from BRCA2 carriers show wild-type chromosome deletions on 13q12-q13. Cancer Res 1995; 55: 4830-4832.

[11] Fan SJ, Yuan RQ, Ma YX, et al. Mutant BRCA1 genes antagonize phenotype of wild-type BRCA1. Oncogene 2001; 20: 8215-35.

[12] Khoo US, Ozcelik H, Cheung ANY, et al. Somatic mutations in the BRCA1 gene in Chinese sporadic breast and ovarian cancer. Oncogene 1999; 18: 4643-6.

[13] Signori E, Bagni C, Papa S, et al. A somatic mutation in the 5' UTR of BRCA1 gene in sporadic breast cancer causes downmodulation of translation efficiency. Oncogene 2001; 20: 4596600 .

[14] Thompson ME, Jensen RA, Obermiller PS, et al. Decreased expression of BRCA1 accelerates growth and is often present during sporadic breast-cancer progression. Nat Genet 1995; 9:444-50.

[15] Wilson CA, Ramos L, Villasenor MR, et al. Localization of human BRCA1 and its loss in high-grade, non-inherited breast carcinomas. Nat Genet 1999; 21: 236-40.

[16] Catteau A, Harris WH, Xu CF, Solomon E. Methylation of the BRCA1 promoter region in sporadic breast and ovarian cancer: correlation with disease characteristics. Oncogene 1999; 18: 1957-65.

[17] Birgisdottir V, Stefansson OA, Bodvarsdottir SK, et al. Epigenetic silencing and deletion of the BRCA1 gene in sporadic cancer Breast Cancer Res 2006; 8: R38.

[18] Bergthorsson JT, Jonasdottir A, Johannesdottir G, et al. Identification of a novel splice-site mutation of the BRCA1 gene in two breast cancer families; Screening reveals low frequency in Icelandic breast cancer patients. Human Mutat 1998; (Suppl 1): S195-7.

[19] Johannesdottir G, Gudmundsson J, Bergthorsson JT, et al. High prevalence of the 999del5 mutation in Icelandic breast and ovarian cancer patients. Cancer Res 1996; 56: 3663-5.

[20] Thorlacius S. Sigurdsson S, Bjarnadottir H, et al. Study of a single BRCA2 mutation with high carrier frequency in a small population. Am J Hum Genet 1997; 60: 1079-84.

[21] Tulinius H, Olafsdotir GH, Sigvaldason H, et al. The effect of a single BRCA2 mutation on cancer in Iceland. J Med Genet 2002; 39: 457-62.

[22] Thorlacius S, Struewing JP, Hartge P, et al. Population-based study of risk of breast cancer in carriers of BRCA2 mutation. Lancet 1998; 352: 1337-9.

[23] Thorlacius S, Olafsdottir G, Tryggvadottir L, et al. A single BRCA2 mutation in male and female breast cancer families from Iceland with varied cancer phenotypes. Nat Genet 1996; 13: 117-9.

[24] Tryggvadottir L, Olafsdottir EJ, Gudlaugsdottir S, et al. BRCA2 mutation carriers, reproductive factors and breast cancer risk. Breast Cancer Res 2003; 5: R121-28.

[25] Rafnar T, Benediktsdottir KR, Eldon BJ, et al. BRCA2, but not BRCA1, mutations account for familial ovarian cancer in Iceland: a population-based study. Eur J Cancer 2004; 40: 2788-93.

[26] Tryggvadottir L, Sigvaldason H, Olafsdottir GH, et al. Populationbased study of changing breast cancer risk in Icelandic BRCA2 mutation carriers, 1920-2000. J Natl Cancer Inst 2006; 98: 116-22.

[27] Tryggvadottir L, Vidarsdottir L, Thorgeirsson T, et al. Prostate cancer progression and survival in BRCA2 mutation carriers. J Nat Cancer Inst 2007; 99: 929-35.

[28] Ingvarsson S, Sigbjornsdottir BI, Huiping C, et al. Mutation analysis of the CHK2 gene in breast carcinoma and other cancers. Breast Cancer Res 2002; 4: R4.

[29] Stacey SN, Sulem P, Johannsson OT, et al. The BARD1 Cys557Ser variant and breast cancer risk in Iceland. PLoS Med 2006; 3: 1103 13.

[30] Vidarsdottir L, Bodvarsdottir SK, Hilmarsdottir H, et al. Breast cancer risk associated with AURKA 91T->A polymorphism in relation to BRCA mutations. Cancer Lett 2007; 250: 206-12.

[31] Breast Cancer Linkage Consortium. Pathology of familial breast cancer: differences between breast cancers in carriers of BRCA1 or BRCA2 mutations and sporadic cases. Lancet 1997; 349: 1505-10.

[32] Marcus JN, Watson P, Page DL, et al. Hereditary breast cancer: pathobiology, prognosis, and BRCA1 and BRCA2 gene linkage. Cancer 1996; 77: 697-709.

[33] Johannsson OT, Idvall I, Anderson C, et al. Tumor biological features of BRCA1-induced breast and ovarian cancer. Eur J Cancer 1997; 33: 362-71.

[34] Ingvarsson S, Geirsdottir EK, Johannesdottir G, et al. High incidence of loss of heterozygosity in breast tumours from carriers of the 999del5 BRCA2 mutation. Cancer Res 1998; 58, 4421-5.

[35] Tirkkonen M, Johannsson O, Agnarsson BA, et al. Distinct somatic genetic changes associated with tumour progression in carriers of BRCA1 and BRCA2 germ-line mutations. Cancer Res 1997; 57: 1222-7.

[36] Hedenfalk I, Duggan D, Chen Y. Gene-expression profiles in hereditary breast cancer. Adv Anat Pathol 2002; 9: 1-4.

[37] Ouchi M, Fujiuchi N, Sasai K, et al. BRCA1 phosphorylation by Aurora-A in the regulation of $\mathrm{G}(2)$ to $\mathrm{M}$ transition. $\mathrm{J}$ Biol Chem 2004; 279: 19643-8.

[38] Sankaran S, Crone DE, Palazzo RE, Parvin JD. Aurora-A kinase regulates breast cancer-associated gene 1 inhibition of centrosomedependent microtubule nucleation Cancer Res 2007; 67: 11186-94. 
[39] Bodvarsdottir SK, Hilmarsdottir H, Birgisdottir V, et al. AuroraA amplification associated with BRCA2 mutation in breast tumours. Cancer Lett 2007; 248: 96-102.

[40] Zhou HY, Kuang J, Zhong L, et al. Tumour amplified kinase STK15/BTAK induces centrosome amplification, aneuploidy and transformation. Nat Genet 1998; 20: 189-93.

[41] Tutt A, Gabriel A, Bertwistle, et al. Absence of Brca2 causes genomic instability by chromosome breakage and loss associated with centrosome amplification. Curr Biol 1999; 9: 1107-10.

[42] Olivier M, Hainaut P. TP53 mutation pattern in breast cancers: searching for clues of environmental carcinogenesis. Semin Cancer Biol 2001; 11: 353-60.

[43] Crook T, Crossland S, Crompton MR, et al. p53 mutations in BRCA1-associated familial breast cancer. Lancet 1997; 350: 638-9.

[44] Greenblatt MS, Chappuis PO, Bond JP, et al. TP53 mutations in breast cancer associated with BRCA1 or BRCA2 germ-line mutations. Cancer Res 2001; 61: 4092-97.

[45] Sullivan A, Yuille M, Repellin C, et al. Concomitant inactivation of p53 and Chk2 in breast cancer. Oncogene 2002; 21: 1316-24.

[46] Eiriksdottir G, Barkardottir RB, Agnarsson BA, et al. High incidence of loss of heterozygosity at chromosome $17 \mathrm{p} 13$ in breast s from BRCA2 mutation carriers. Oncogene 1998; 16: 216.

[47] Gretarsdottir S, Thorlacius S, Valgardsdottir R, et al. BRCA2 and p53 mutations in primary breast cancer in relation to genetic instability. Cancer Res 1998; 58: 859-62.

[48] Lee H, Trainer AH, Friedman LS, et al. Mitotic checkpoint inactivation fosters transformation in cells lacking the breast cancer susceptibility gene, BRCA2. Mol Cell 1999; 4: 1-10.

[49] Ingvarsson S. Tumor suppressor genes on human chromosome 3 and cancer pathogenesis. Cancer Genomics Proteomics 2005; 2 : 247-54.

[50] Ingvarsson S, Agnarsson BA, Sigbjornsdottir BI, et al. Reduced Fhit expression in sporadic and BRCA2-linked breast carcinomas. Cancer Res 1999; 59; 2682-9.
[51] Ingvarsson S. FHIT alterations in breast cancer. Semin Cancer Biol 2001; 11:361-6.

[52] Weiske J, Albring KF, Huber O. The tumor suppressor Fhit acts as a repressor of $\beta$-catenin transcriptional activity. Proc Natl Acad Sci USA 2007; 104: 20344-9.

[53] Sigbjörnsdottir BI, Ragnarsson G, Agnarsson BA, et al. Chromosome 8p alterations in sporadic and BRCA2 999del5 linked breast cancer. J Med Genet 2000; 37: 342-7.

[54] Bell DW, Varley JM, Szydlo TE, et al. (1999) Heterozygous germ line hCHK2 mutations in Li-Fraumeni syndrome. Science 1999; 286: 2528-31.

[55] Meijers-Heijboer H, Van Den Ouweland A, Klijn J, et al. Lowpenetrance susceptibility to breast cancer due to CHEK2 $(*) 1100 \mathrm{delC}$ in noncarriers of BRCA1 or BRCA2 mutations. Nat Genet 2002; 31: $55-9$.

[56] Ko MJ, Murata K, Hwang DS, Parvin JD. Inhibition of BRCA1 in breast cell lines causes the centrosome duplication cycle to be disconnected from the cell cycle. Oncogene 2006; 25: 298-303.

[57] Starita LM, Machida Y, Sankaran S, et al. BRCA1-dependent ubiquitination of gamma-tubulin regulates centrosome number. Mol Cell Biol 2004; 24: 8457-66.

[58] Thai TH, Du F, Tsan JT, et al. Mutations in the BRCA1-associated RING domain (BARD1) gene in primary breast, ovarian and uterine cancers. Hum Mol Genet 1998; 7: 195-202.

[59] Ghimenti C, Sensi E, Presciuttini S, et al. Germline mutations of the BRCA1-associated ring domain (BARD1) gene in breast and breast/ovarian families negative for BRCA1 and BRCA2 alterations. Genes Chrom Cancer 2002; 33: 235-42.

[60] McCarthy EE, Celebi JT, Baer R, Ludwig T. Loss of Bard1, the heterodimeric partner of the Brcal tumor suppressor, results in early embryonic lethality and chromosomal instability. Mol Cell Biol 2003; 23: 5056-63.

[61] Stacey SN, Manolescu A, Sulem P, et al. Common variants on chromosomes $2 \mathrm{q} 35$ and $16 \mathrm{q} 12$ confer susceptibility to estrogen receptor-positive breast cancer. Nat Genet 2007; 39: 865-9.

(c) Sigurdur Ingvarsson; Licensee Bentham Open .

This is an open access article licensed under the terms of the Creative Commons Attribution Non-Commercial License (http://creativecommons.org/licenses/by-

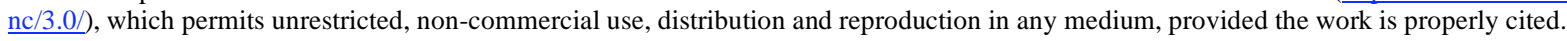

